\title{
Situation and Strategy of Yang Style Tai Chi in Handan
}

\author{
Shiru Hao \\ Department of Physical Education in Baoding College \\ Weilong $\mathrm{Xu}$ \\ Department of Physical Education in Handan College
}

\begin{abstract}
Tai chi has became one the most famous sports to keep fit for its prominent fitness effect and profound culture deposit. Among all kinds of styles, Yang-style Tai chi spreads most widely. Handan have high representative for it's the place of origin for Yang style Tai chi. This article choose the method of documental, observation, questionnaire survey, conversational, mathematical statistics to make analysis on current situation of Yang style Tai chi practitioners. Also made a deep analysis on problems when popularize Yang style Tai chi, and give strategies to them. Improve the comprehension to Tai chi and the correct way to use it in purpose of give a scientific theoretical basis to popularize of Yang style Tai chi.
\end{abstract}

KEYWORD: Handan city; Yang style Tai chi; Current situation; Strategy

\section{INTRODUCTION}

As the standard of living keep increasing, concept of health preserving become more and more popular among people, thus make Tai chi, the non-material culture inheritance become more and more popular among people. After investigation, we found 5 genres of Yang style Tai chi are the most famous ones and been widely circulated. Yongnian, Handan have high representative for it's the place of origin for Yang style Tai chi. The current situation of Yang style Tai chi can be gain by doing investigation on practitioners. The information of Tai chi is not insufficient when searching documents and datum, most of them are the theory and health preservation knowledge about how Tai chi works when elderly people practice it, but rarely find some researching reference to generalization, even nothing on current situation of Yang style Tai chi. This article made a deep analysis on problems exist in Tai chi generalization by doing investigation on the place of origin for Yang style Tai chi, and give relevant strategy to the development in order to improve the cognition of the theory of Tai chi and the correct way to practice it. Aimed at give more theoretical basis and reference to the generalization and development of Yang style Tai chi.

\section{OBJECT AND METHOD}

\subsection{Object}

Practitioner of Yang style Tai chi in Handan.

\subsection{Method}

\subsubsection{Methods of documentation}

After consult documents in Chinese Academic Journal and Chinese Doctor \& Master thesis database by visiting CNKI and school library, informations about current situation of popularize for Yang style Tai chi had been collected and organized to be a theoretical basis of this article.

\subsubsection{Observation}

Observe the practitioner of Tai chi, comprehend and record their gender rate, age, account of practitioner and other objective data.

\subsubsection{Questionnaire method}

Aimed at current situation of Tai chi, questionnaire had been made to leaders in Sports Bureau, descendant and master of Tai chi, and practitioner at each practice site. Efficient retrieve rate is $99 \%$.

\subsubsection{Conversational}

After made conversation with professionals and staff in Sports Bureau, viewpoints for current situation of 
Tai chi and its development process gained as the important reference basis of questionnaire design.

\subsubsection{Mathematical statistics}

Import the datum to EXCEL system, use SPSS13.0 to make statistics and analysis.

\section{PURPOSE AND SIGNIFICANCE}

After make deep analysis on problems exist in development of Yang style Tai chi, give reference strategy on problem solution and development, improve the cognition to the theory and correct way to practice it. Aim at give a scientific theoretical basis. Meanwhile, enlarge the influence and national prestige of Yang style Tai chi.

\section{RESULT ANALYSIS}

\subsection{Situation of practitioners}

Result of investigation: figure 1 shows the gender of practitioners, female are much more than male ones. The reason could be: male people usually lack of patience; female people usually need to improve their physical condition, and have more leisure time than male people; Male people usually busy on family livelihood and working stuff.

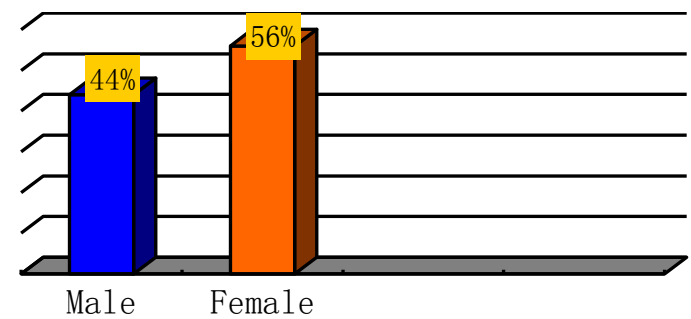

Figure 1 gender of practice

Figure 2 shows: $88 \%$ of the practitioners tend to practice in park rather than in professional stadium. The reason are: parks are large and have a nice environment with clean air which make people feel cheerful when practice, some of the park also have fitness facilities which can give a better practice atmosphere. Most of the practitioners are elderly people, communication is easy among them which is good to improve the interest of Tai chi practice; There always have some Tai chi club in the park, with the organization and management, expert organizer and instructor, communication on practicing, people can easily improve their relationships and the skill of Tai chi practice. The reason of there's not so many people choose professional stadium is because of the consumption level of the practitioner still need a improvement.

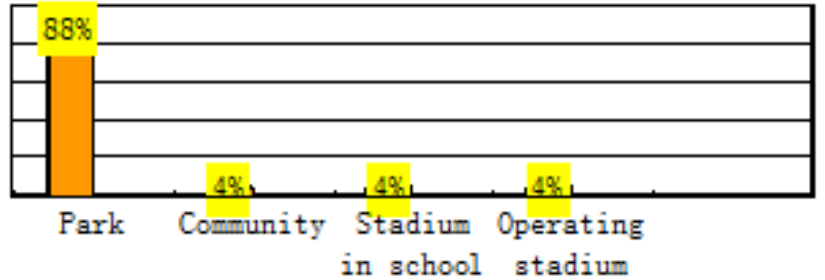

Figure 2 place of practice

Figure 3 shows: most of the practitioner choose books and $\mathrm{CD}$ as the approaches of learning, only few of them learn Tai chi by teaching from a master, and this is not good for the popularize of Yang style Tai chi, also shows a insufficient in professional coach talents.

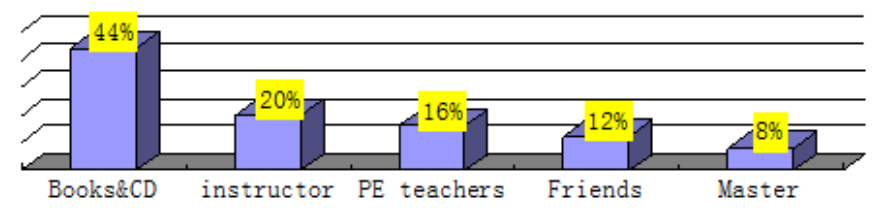

Figure 3 approach of practice

\subsection{Age of practitioners}

Take the WHO age segmentation as reference, there are 4 age segmentation under 17years old(Minor), 18-44 years old(Youth), 45-49years old(Midlife), over 60years old(Agedness)

Age distribution showed by figure 4 is: $56 \%$ are people who between $45-49$ years old, $28 \%$ are over 60 years old, $18-44$ take $16 \%$ of all, $0 \%$ taken by people who under 17 years old. Most of the practitioners are middle aged or elderly people who already retired, which means when people step in middle life, physical decline appears, replenishment is necessary. On the other hand, a small amount of youth practitioner show a lack of reserve force for Yang style Tai chi.
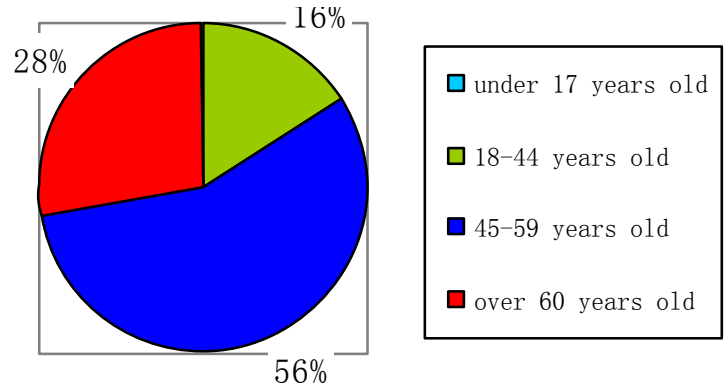

Figure 4 age of practitioners

\subsection{Psychological characteristics of practitioners}

Figure 5 shows $55 \%$ of the practitioners' purpose is keeping fit, then $25 \%$ for entertainment, interest take $20 \%$, mandatory requirements take $2 \%$. This means most of them are aware of the important of fitness, which is good for improvement of the development for Yang style Tai chi. As a lot of elderly people's 
life is dull and lonely, practice Tai chi can help making their life plentiful, and enlarge their contacts circle, combine body building and entertainment make the most of practitioners are elderly people.

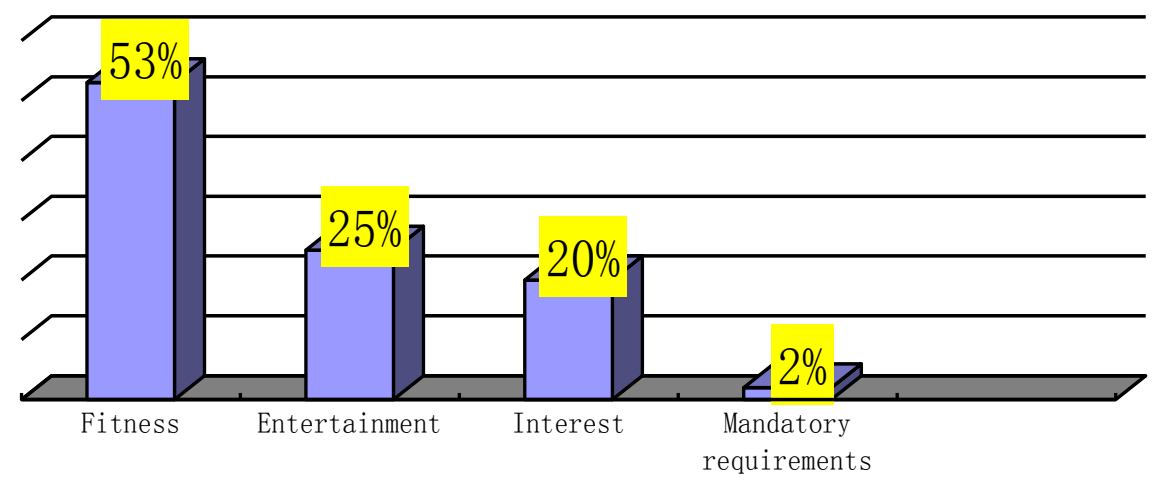

Figure 5 Psychological characteristics of practitioners

\subsection{Physiological characteristic of practitioners}

Investigation shows: most of the practitioners are elderly people. Physical function and immunity decrease as age increases which make people easy to falling ill, meanwhile, physical load-bearing capacity of the body become weaken, too. All those show that people at this age can only afford general practice, and hard to raise the exercise load.

\section{CONCLUSION AND SUGGESTION}

\subsection{Conclusions}

(1) Most of the practitioners are female

(2) Backup talent are insufficient

(3) Professional trainer and political instructor are insufficient

\subsection{Suggestions}

(1) Make commercial packing for Yang style Tai chi, improve the cognition for Tai chi, hold sports and communication events to improve the enthusiasm for Tai chi practice.
(2) Publishes policy to organize the professional training from Tai chi master to physical education teachers in school, make Tai chi a general component of physical education to form a virtuous cycle in order to solve the problem of insufficient in reserve force.

(3) Train more professional teachers and instructor, transform the traditional folk inherit to college spreading.

\section{REFERENCES}

[1] WHO age segmentation, http://www.baidu.com

[2] Wenhan Wu, Development of Tai chi in recent century 2005.7, http://www.hdtaiji.com

[3] Yunfang Long Chenglie Zhan Chengzhi Tang, Influence on sleeping and spirit of elderly people by Tai chi, 2000.4, "Occupational health and disease".

[4] Guosheng Zhang, Discussion on history, current situation and development of Handan Tai chi, Baidu Blog of Tai chi Wangluo.

[5] Nantian Yan, History and spread of Yongnian Tai chi, 2006.4.1, http://www.21wulin.com 